\title{
Photoacoustic microscopy enables multilayered histological imaging of human breast cancer without staining
}

Terence T. W. Wong, Ruiying Zhang, Pengfei Hai, Rebecca L. Aft, Deborah V. Novack, et al.

Terence T. W. Wong, Ruiying Zhang, Pengfei Hai, Rebecca L. Aft, Deborah V. Novack, Lihong V. Wang, "Photoacoustic microscopy enables multilayered histological imaging of human breast cancer without staining," Proc. SPIE 10494, Photons Plus Ultrasound: Imaging and Sensing 2018, 1049443 (19 February 2018); doi: 10.1117/12.2291008

SPIE. Event: SPIE BiOS, 2018, San Francisco, California, United States 


\title{
Photoacoustic microscopy enables multilayered histological imaging of human breast cancer without staining \\ Terence T. W. Wong ${ }^{\mathrm{a}, \mathrm{b}}$, Ruiying Zhang ${ }^{\mathrm{b}}$, Pengfei Hai ${ }^{\mathrm{a}, \mathrm{b}}$, Rebecca L. Aft ${ }^{\mathrm{c}, \mathrm{d}}$, Deborah V. Novack ${ }^{\mathrm{e}, \mathrm{f}}$, and Lihong V. Wang, ${ }^{\mathrm{a}, *}$ \\ ${ }^{a}$ Caltech Optical Imaging Laboratory, Andrew and Peggy Cherng Department of Medical \\ Engineering, Department of Electrical Engineering, California Institute of Technology, Pasadena, CA 91125, USA; \\ ${ }^{\mathrm{b}}$ Optical Imaging Laboratory, Department of Biomedical Engineering, Washington University in St. Louis, St. Louis, MO 63130, USA; \\ 'Department of Surgery, Washington University School of Medicine, St. Louis, Missouri 63110, USA; \\ ${ }^{\mathrm{d} J o h n}$ Cochran Veterans Hospital, St. Louis, Missouri 63106, USA; \\ ${ }^{e}$ Musculoskeletal Research Center, Division of Bone and Mineral Diseases, Department of \\ Medicine, Washington University School of Medicine, St. Louis, Missouri 63110, USA; \\ ${ }^{\mathrm{f}}$ Department of Pathology, Washington University School of Medicine, St. Louis, Missouri 63110, USA
}

\begin{abstract}
In 2016, an estimated 250,000 new cases of invasive and non-invasive breast cancer were diagnosed in US women. About $60-75 \%$ of these cases were treated with breast conserving surgery (BCS) as the initial therapy. To reduce the local recurrence rate, the goal of BCS is to excise the tumor with a rim of normal surrounding tissue, so that no cancer cells remain at the cut margin, while preserving as much normal breast tissue as possible. Therefore, patients with remaining cancer cells at the cut margin commonly require a second surgical procedure to obtain clear margins. Different approaches have been used to decrease the positive margin rate to avoid re-excision. However, these techniques are variously ineffective in reducing the re-operative rate, difficult to master by surgeons, or time-consuming for large specimens. Thus, 20-60\% of patients undergoing BCS still require second surgeries due to positive surgical margins. The ideal tool for margin assessment would provide the same information as histological analysis, without the need for processing specimens. To achieve this goal, we have developed and refined label-free photoacoustic microscopy (PAM) for breast specimens. Exploiting the intrinsic optical contrast of tissue, ultraviolet (UV) laser illumination can highlight cell nuclei, thus providing the same contrast as hematoxylin labeling used in conventional histology and measuring features related to the histological landscape without the need for labels. We demonstrate that our UV-PAM system can provide label-free, high-resolution, and histology-like imaging of fixed, unprocessed breast tissue.
\end{abstract}

Keywords: Cancer, translational research, photoacoustic imaging, microscopy, label-free, histology

\section{INTRODUCTION}

Breast cancer is the most common cancer diagnosed in women. In 2016, an estimated 250,000 new cases of invasive breast and non-invasive breast cancer were diagnosed in US women ${ }^{1}$. To reduce the local recurrence rate, $60-75 \%$ of these cases were treated with breast conserving surgery $(\mathrm{BCS})^{1}$. BCS is defined as a lumpectomy followed by radiation therapy. Removal of the entire cancer is best ensured by performing histopathology and observing only normal breast tissue at the surgical margins, which is the current standard of care (SOC). Failure to achieve appropriate margins at the initial operation requires a second surgical procedure ${ }^{2}$. These re-excision surgeries are a burden to patients financially, physically, and psychologically, result in increased complications, and can delay recommended adjuvant therapies.

Complete cancer removal can only be verified by post-operative pathology. The gold standard for microscopic margin assessment is examination of post-operative histopathology sections. To get these sections, the preparation

Photons Plus Ultrasound: Imaging and Sensing 2018, edited by Alexander A. Oraevsky, Lihong V. Wang, Proc. of SPIE Vol. 10494, 1049443 · @ 2018 SPIE · CCC code: 1605-7422/18/\$18 · doi: 10.1117/12.2291008 
procedure can take up to hours and days, hindering the possibility of providing real-time feedbacks to surgeons for better patient outcome. Intraoperatively, histological examination of tissue can be performed by frozen sectioning, however, which is limited by the difficulties of freezing adipose-rich tissue, such as breast specimens. To address this critical need, we have developed and optimized our UV-PAM for breast tissue imaging. UV laser illumination has an advantage of highlighting cell nuclei, thus providing similar contrast as hematoxylin labeling used in conventional histology. Together with an acoustically defined axial resolution, UV-PAM can provide multilayered histology-like images of the surface and subsurface of breast tissue without physical sectioning, which could provide additional information to pathologists for more accurate diagnosis.

\section{METHODOLOGY}

\section{Ultraviolet photoacoustic microscopy (UV-PAM)}

Our UV-PAM system (Fig. 1), which can handle specimens of various thicknesses, produces images by detecting acoustic waves generated by laser-induced rapid thermoelastic expansion ${ }^{3}$. A focused ultrasonic transducer detects the one-dimensional (1D) depth-resolved PA signals (A-lines). Cross-sectional images (B-scans) or volumetric images (Cscans) can be produced respectively by linear or raster scanning using a motorized scanning stage. The UV-PAM system records and displays the PA B-scan images in real time during data acquisition. Because different absorbers intrinsically possess different optical absorption spectra, when one absorber dominates at a chosen wavelength, a single-wavelength laser can be used to probe a specific biological component without resorting to exogenous labels. Therefore, UV light $(266 \mathrm{~nm})$, which DNA and RNA in cell nuclei absorb by an order of magnitude more strongly than other biological components, is used for illumination in our imaging system ${ }^{4,5}$. At this short wavelength, high spatial resolution can also be achieved. The lateral imaging resolution was experimentally measured to be $\sim 330 \mathrm{~nm}^{4}$, comparable to that of a conventional bright-field optical microscope with a high numerical aperture (NA) and sufficient to identify individual cell nuclei. Hence, UV-PAM images should be able to reveal individual cell nuclear information without labeling.

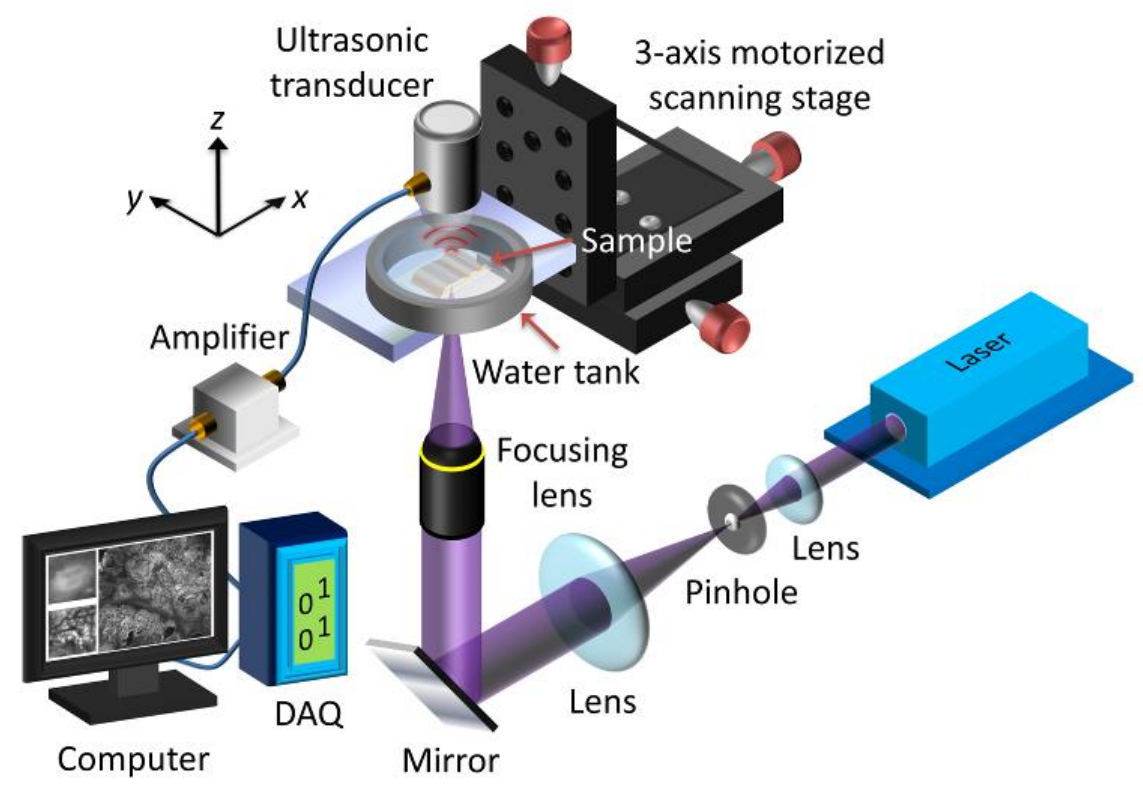

Figure 1. Schematic of the UV-PAM system for surgical margin imaging. The UV laser beam is spatially filtered and expanded by a pair of lenses and a pinhole. The beam is then focused by a focusing lens and illuminated onto the bottom of the breast tissue specimen (Sample). The forward propagating acoustic waves are detected by a focused ultrasonic transducer. The received acoustic pressure is transduced into an electric signal, amplified, and recorded by a data acquisition (DAQ). 


\section{RESULTS}

\section{Label-free UV-PAM of fixed, unprocessed human breast tumors versus traditional microscopy of specimens processed with standard histology}

To show the full potential of UV-PAM, we imaged formalin-fixed but unprocessed human breast tumors (Figs. 2 and 3). After surgical excision, the breast tumors were fixed in formalin to prevent tissue degradation. Small samples were taken from the tumor/normal interface and sent for PA imaging. Subsequently the specimens were processed according to standard tissue preparation protocol (i.e., paraffin embedding, sectioning, and H\&E staining). Sections corresponding to the UV-PAM images were analyzed by conventional bright-field optical microscopy. Since the UV-PAM system images the surface and subsurface of a specimen (Fig. 4), to prepare the best corresponding H\&E-stained images, superficial sections were utilized. These H\&E-stained sections did not exactly replicate the surface imaged by UV-PAM, but the image features are still remarkably similar.

For the fixed, unprocessed breast tumor specimen from the first patient, the region of interest (ROI) was $10.0 \mathrm{x}$ $4.2 \mathrm{~mm}^{2}$ (Fig. 2a). Fig. 2b shows a UV-PAM image of the ROI. The specimen was put in a lab-made sample holder, which flattened the tissue for wide field-of-view (FOV) in-focus imaging. After PA imaging, the corresponding sections were prepared histologically and images were acquired (Fig. 2c). The close-up UV-PAM images (Fig. 2d and f) reveal features similar to their corresponding H\&E-stained images (Fig. 2e and g). Each image centers on expanded breast ducts with a cribriform pattern of cellular proliferation, characteristic of ductal carcinoma in situ, that can be readily discerned in both the UV-PAM and H\&E-stained images. Smaller nests of densely packed nuclei, representing invasive ductal carcinoma, can also be seen along the top edge in Fig. 2d-g).

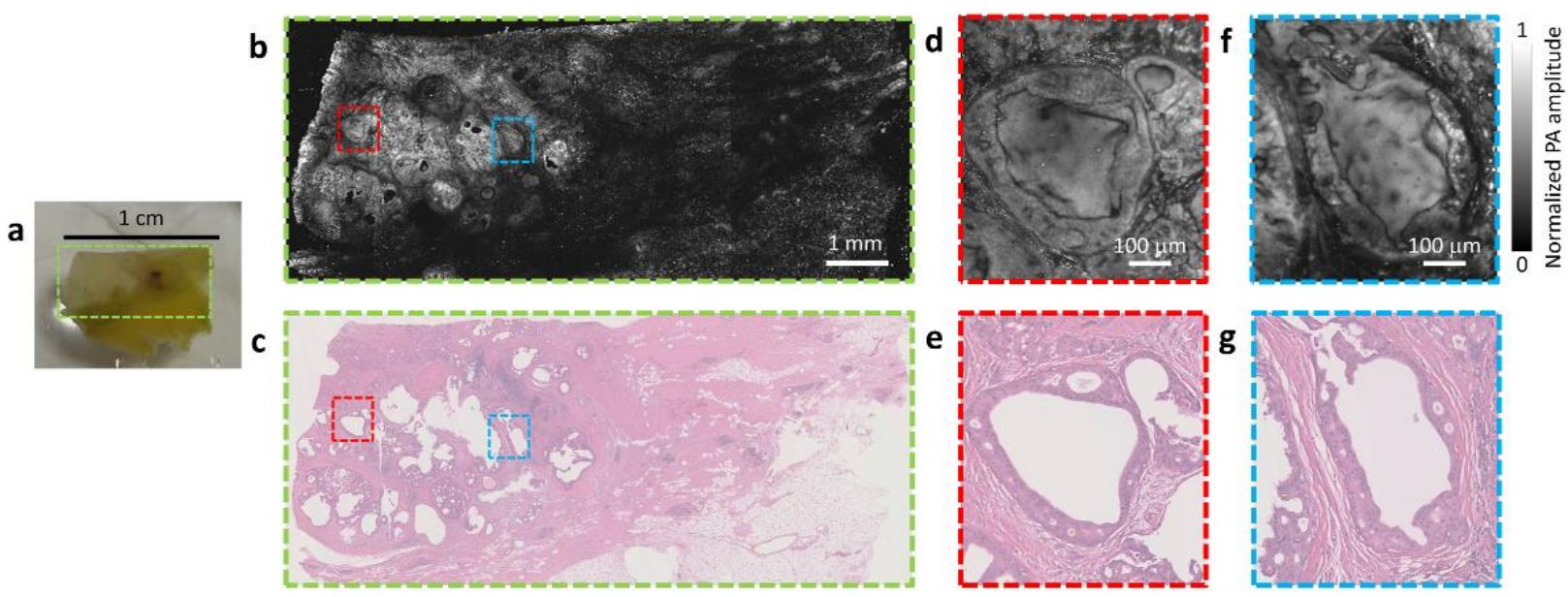

Figure 2. Imaging of a breast tumor from the first patient. (a) Photograph of the first breast tissue specimen. (b and c) UVPAM and H\&E-stained images of the fixed, unprocessed breast tumor of the green dashed region in (a), respectively. Note that the H\&E-stained histologic image was acquired after processing, sectioning, and staining the excised breast tissue. (d and e) Zoomed-in UV-PAM and H\&E-stained images of the red dashed regions in (b) and (c), respectively. (f and $\mathbf{g}$ ) Zoomed-in UV-PAM and H\&E-stained images of the blue dashed regions in (b) and (c), respectively.

For the second patient, a ROI of $7.5 \times 4.5 \mathrm{~mm}^{2}$ (Fig. 3a) was imaged by our UV-PAM system (Fig. 3b). The corresponding H\&E-stained image is shown in Fig. 3c. The close-up UV-PAM image (Fig. 3d) shows a number of small bright cell nuclei that correspond to lymphocytes on the histology image (Fig. 3e). Lymphocytes are often associated with cancer. Comparing the two close-up images (Fig. 3d and e), we observe denser lymphocytes in our UV-PAM image. This difference can be explained by the greater imaging penetration depth of UV-PAM $(\sim 100 \mu \mathrm{m})$ in comparison to the sectioned slice thickness $(\sim 7 \mu \mathrm{m})$ of the H\&E-stained tissue. When the cell nuclei are densely packed along the lateral and axial directions, individual cell nuclei cannot be resolved, resulting in a bright and dense feature, as seen in another close-up UV-PAM image (Fig. 3f). In this case, the corresponding H\&E-stained image (Fig. 3g) demonstrates densely packed breast tumor cells. 
a

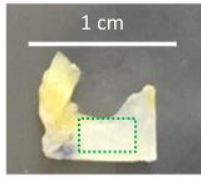

b

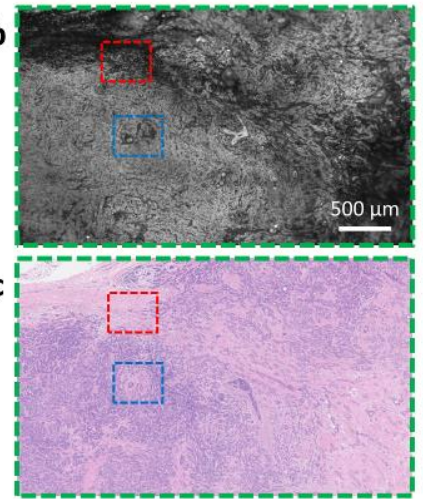

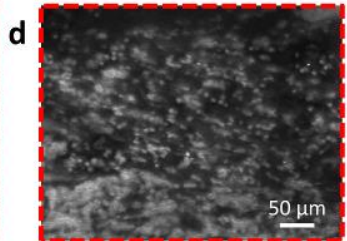

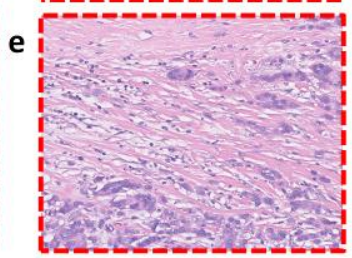

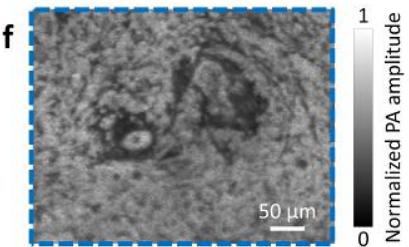

g

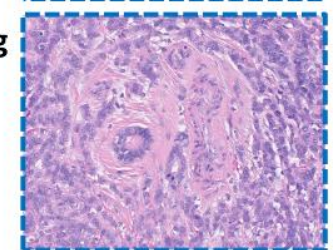

Figure 3. Imaging of a breast tumor from the second patient. (a) Photograph of the second breast tissue specimen. (b and c) UV-PAM and H\&E-stained images of the fixed, unprocessed breast tumor of the green dashed region in (a), respectively. (d and e) Zoomed-in UV-PAM and H\&E-stained images of the red dashed regions in (b) and (c), respectively. (f and g) Zoomed-in UV-PAM and H\&E-stained images of the blue dashed regions in (b) and (c), respectively.

For a third patient sample, the ROI was $7.8 \times 3.0 \mathrm{~mm}^{2}$ (Fig. 4a). As with the second patient, in our UV-PAM image we can see a bright signal in the top region (Fig. 4b). This region also represents a high-grade tumor. With the high resolution provided by UV-PAM and without any sample processing, UV-PAM can also image fat cells, which are clearly shown with the zoomed-in UV-PAM images (Fig. 4c and d). In Fig. 4d, we can easily identify the cell nuclei of the fat cells, which are normally dissolved during the standard tissue preparation protocol.
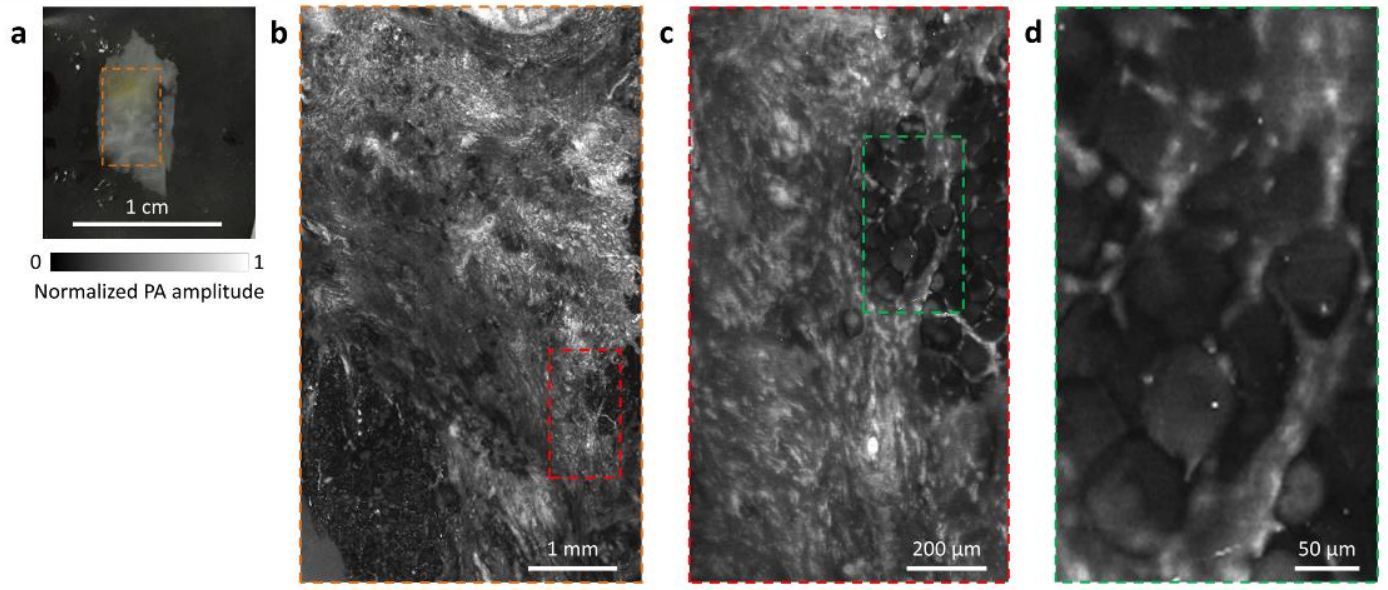

Figure 4. Imaging of a breast tumor from the third patient. (a) Photograph of the third breast tissue specimen. (b) UV-PAM image of the fixed, unprocessed breast tumor of the orange dashed region in (a). (c) Zoomed-in UV-PAM image of the red dashed regions in (b). (d) Zoomed-in UV-PAM image of the green dashed regions in (c).

To further demonstrate the unique advantage of UV-PAM, which is to generate multilayered histology-like images from a single raster scan, we time-gated our measured PA signals to generate depth-resolved images of the first patient's breast tumor specimen (Fig. 5). Fig. 5a shows the entire field-of-view at 8 different depths with an axial step size of $22 \mu \mathrm{m}$. Two close-up UV-PAM images of the red and blue dashed boxes (marked in Fig. 5a) are also shown with the 8 corresponding depths (Fig. $5 \mathrm{~b}$ and $\mathrm{c}$ ). This multilayered imaging capability does not come at the expense of scanning time as the time-of-flight information of the PA waves automatically provide the depth information of the specimens. This multilayered imaging feature maximizes the chance of identifying tumor cells of an excised tissue, which cannot be provided by bright-field optical microscopy. 


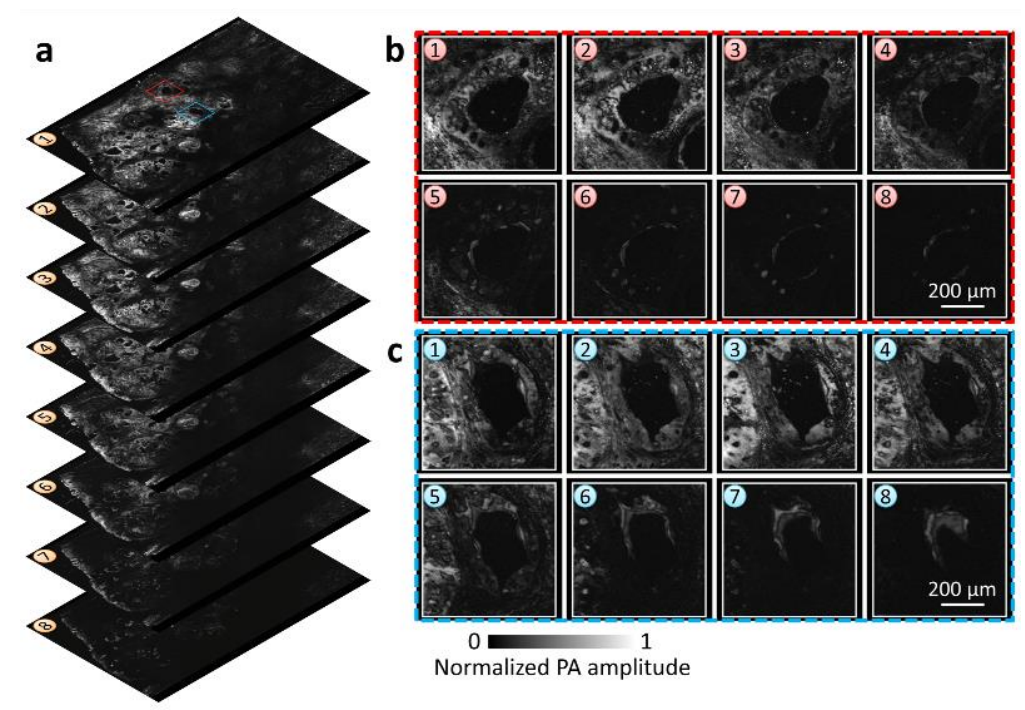

Figure 5. Series of depth-resolved images of the first patient's breast tumor specimen imaged by UV-PAM. The depthresolved images are shown at 8 depths with an axial step size of $22 \mu \mathrm{m}$.

\section{CONCLUSIONS}

To conclude, we have demonstrated and optimized UV-PAM for breast tissue imaging, providing images similar to that can be seen on H\&E-stained slide without labels. With the high resolution images provided by UV-PAM, diagnostic features, including cell nuclear sizes and internuclear distance can also be measured. These features are important for cytologic and histologic grade. In the future, these useful parameters could be provided to automatic algorithms and recognize malignancy in UV-PAM images alone. While we have concentrated on breast tissue in this study, UV-PAM can be optimized for other cancer specimens where margin status is critical for patient outcome.

\section{REFERENCES}

[1] American Cancer Society, Breast Cancer Facts \& Figures 2016-2017., Am. Cancer Soc. Inc, Abstract (2016).

[2] Clarke, M. et al. "Effects of radiotherapy and of differences in the extent of surgery for early breast cancer on local recurrence and 15-year survival: an overview of the randomised trials," Lancet 366, 2087-2106, (2005).

[3] Wang, L. V., and Yao J., "A practical guide to photoacoustic tomography in the life sciences," Nat. Methods 13, 627-638 (2016).

[4] Wong, T. T. W. et al. "Fast Label-free multilayered histology-like imaging of human breast cancer by photoacoustic microscopy," Sci. Adv. 3, e1602168 (2017).

[5] Wong, T. T. W. et al. "Label-free automated three-dimensional imaging of whole organs by microtomy-assisted photoacoustic microscopy," Nat. Commun. 8, 1386 (2017). 